\title{
The Level of Quality of Life among Individuals who Stutter in Jordan and Its Relationship with Some Demographic Variables
}

\author{
Dr. Noor Talal ALBdour ${ }^{*} \quad$ Dr. Yahya Al-Dhaimat ${ }^{2} \quad$ Mohammad A. Damhoureyeh ${ }^{3}$ \\ 1.Department of Special Education, faculty of Education, Al-Hussein Bin Talal University PO 20, Jordan \\ 2.Department of Special Education, faculty of Education, Al-Hussein Bin Talal University, Jordan \\ 3.Department of Hearing and Speech Sciences, School of Rehabilitation Sciences, the University of Jordan, \\ Amman, Jordan
}

\begin{abstract}
The current study aimed to identify the level of quality of life among Individuals who Stutter in Jordan and its relationship to some demographic variables using the correlative descriptive approach. In order to achieve the objectives of the study, the researchers used the quality of life scale-BRIEF-WHOQQL, prepared by the World Health Organization (WHO), on a sample $(\mathrm{N}=32)$ males and females Stutters. The results of the study showed that the level of quality of life among individual who stutters in Jordan was low, as the physical health field ranked first with the lowest level in the quality of life followed by the field of social relations and then the mental health and finally the field of environment. They also showed that there were no statistically significant differences in the level of quality of life among Individuals who Stutter in Jordan attributed to the variables of age, gender, marital status, family income and the degree of stuttering severity. The study concluded with a set of research and educational recommendations.
\end{abstract}

Keywords: Demographic variables, Individuals who stutter, Quality of life.

DOI: $10.7176 / \mathrm{JEP} / 11-26-08$

Publication date:September $30^{\text {th }} 2020$

\section{Introduction}

1.1 Stuttering

Since ancient times, stuttering has been known as a phenomenon found among all people at all cultural, social, and economic levels. Moreover, it is one of the most common disorders of fluency, and it is a complex and multidimensional problem that could be observed and heard easily. A Stutter person suffers from psychological adjustment problems and feelings of fear, anxiety, confusion, shame, and social isolation at school or in social situations which affects one's self-esteem. This makes an individual avoid verbal situations least of ridicule, reduce one's adaptation to the surrounding environment and impede his/her implementation of daily life activities. Consequently, all these problems affect individuals' attitudes and the acceptance of their selves and make stuttering a main problem in the quality of life.

Given the concept of fluency and specifically fluency disorders, Starkweather et.al, (1990), Amayerh and Natoor (2014) stated that fluency meant the easily, gently, and effortlessly flow of speech, whereas disfluency disorders - the most common and frequent stuttering — means that speech does not get out easily and that words do not move smoothly.

In 1977, the World Health Organization defined the stuttering as a fluency disorder that infects the flow of speech despite the individual's full knowledge of what s/he would say but is unable to say it because of involuntary repetition, prolongation or cessation. Al-Zureikat (2018) defined it is a disturbance in the natural fluency of speech characterized by repetitions and prolonged hesitations in speech. (Guitar, 2006; Ramig \& Doge, 2010) argued that stuttering is involuntary repetition and prolonged silent pauses before uttering speech which affects the flow of speech. The definition of Wingate (1964) (as cited in Damico. et.al., 2010) is regarded the best to distinguish between the natural disfluency and stuttering. It considers stuttering a disorder in the fluency of speech, characterized by repetitions or prolongation of parts of speech, especially the sounds and syllables accompanied by a strain in the body parts, which indicates a tension related to speech and a general state of excitement, tension and negative feelings such as anger, embarrassment and fear due to the inconsistency in the amount of speech Amayerh and Natoor (2014). Therefore, the researchers define stuttering as a problem in the quality of life, and an abnormal fluency disorder including major symptoms such as repetition, blocks, and prolongation resulting in unseen behaviors such as escaping and avoiding, as well as negative feelings of fear and anxiety during speech.

Al-Dhahir (2010) and Amayerh and Natoor (2014) mentioned a number of preliminary symptoms that appear to Individuals who Stutter, including repetition of sounds, preventing vocal cords from vibrating and speech disruption, prolongation of sounds and secondary behaviors in response to primary behaviors such as eyes winking, head nodding, tension, etc. Whereas the unapparent symptoms include word substitution, deviating from or avoiding discussing a given topic.

The exact causes of stuttering have not been fully known yet, and there are a group of factors, including those related to parents, where (Bannett, 2006; Bloodstein, Bernstem, 2008; Guitar, 2006; Sheehan, 1970; and Van Riper, 
1992) claimed that parents do not cause stuttering but contribute to its development and survival. According to Johnson (1959) and Bennt (2006), parents misperceive the normal disfluency of their children as stuttering parents, in addition to the genetic and developmental factors and the different environment that may cause stuttering. By reviewing many of the factors causing stuttering, researchers found that the most reliable and integral theories in the interpretation of stuttering disorder are those comprehensive theories that take into account neurological, physiological and environmental factors.

Individuals who Stutter usually suffers from emotional and behavioral problems due to the difficulty in speech fluency, and the child may not be aware of the problem at first, but gradually s/he becomes more aware of the speech challenges that are a source of negative feelings, a feeling of discomfort and speech avoidance. Actually, all Individuals who Stutter undergo painful emotional experiences that increase with age they have more fears and anxiety than certain words or situations that provoke their feelings of fear and anxiety, and this is evident clearly through the four-age stages that they go through, summed up by (Al-Bdour, 2013; Al-Khatib et. al., 2018) as follows:

The first stage: Borderline stuttering signs appear in early childhood stage (1.5-5) years, and it has a stuttering rate of 10 times and more and more than two repetitions each time and there are no tension and negative reactions.

The second stage: Beginning stuttering signs appear at the age of (4-6) years in terms of muscle stiffness, rapid repetitions, and some aspects of avoidance, including eye blinking. In addition, there is an evidence suggest the presence of negative attitudes even those young children who stutter (Guttormsen, 2018).

The third stage: Intermediate stuttering signs appear at the age of (6-13) years, where the child is characterized by fear, frustration, and surprise from stuttering. At this stage, there is a frequent block, the avoidance behaviors and stuttering become clear accompanied by the feelings of fear, shame, and embarrassment of stuttering.

The fourth stage: Advanced stuttering which is for adults over 14 years and above. The most common behaviors that characterize this stage are the prolonged blocks and tension. Stuttering to avoid verbal situations of avoidance is lessened and yet accompanied by the strong feelings of fear, embarrassment, shame, negative perception of the self-esteem and the dominance of the negative self-concept.

Al-Khatib et. al. (2018) suggested that Supportive environment conditions surrounding the child including family or school help in achieving auto-recovery. Mainly, stuttering treatment for older children and adolescents is based on direct treatment that focuses on improving feelings, negative attitudes and emotional crises that have developed in a person who suffers from stuttering with the passage of time. It does not only affect the aspects of his psychological life but also his academic achievement, his social and professional life. Al-Zureikat (2018) indicated that the psychological treatment of stuttering focuses on dealing with the factors that cause psychological and emotional problems and the pressures causing stuttering. The specialists focus on the psychological processes and means of defense used, personality development, and controlling feelings of anxiety and fear to reduce stuttering symptoms. Ramig (2010) indicated that speech is a frustrating and confusing experience for a child who Stutter. The strategy of declaration and self-disclosure by parents and listeners contributes to relieving tension during verbal situations and giving the child more spontaneity in speech. This strategy helps convince the child to discuss stuttering and its emotional effects openly and frankly. Van Riper (1992) founded the method of teaching stuttering fluently by regulating the flow of air and reducing the rate of speech velocity, thus, changing the stuttering form to become socially more acceptable and modifying the Individuals who Stutter' negative feelings.

\subsection{Quality of life}

Since we live in an era in which there are many political and economic changes that affect an individual's academic and social life, which increases hardship and pressure and raises the rates of psychological and physical disorders in order to prevent the proper individual compatibility, which affects one's personality, it is necessary to focus on one contemporary and influential aspect in the life of a Individuals who Stutter which is the quality of life. Psychology is considered one of the sciences that cared about the quality of life as a response to scientists' emphasis on the individual's interest in adopting a positive view of life instead of the negative one, as the quality of life is a broad concept in which several aspects such as the subjective and objective aspects of the individual, independence, physical and psychological health, satisfaction with life, family life, social relations, and the relationship of the individual with environment (Bonomi, Patrik Bushnell, 2002; Abu Racine, 2012).

The World Health Organization (WHO) (2013) defined the quality of life as an awareness of the individual's attitude in life in relation to culture and values in which s/he lives and relating it to the individual's own goals, interests and beliefs. It is a broad concept influenced by the individual's psychological and religious condition and his social relationship (Tashtoush \& Al-Qashaar, 2017).

Katlo (2011) also defined the quality of life as the individual's awareness of his/her position in life in relation to the culture and values in which the individual lives. Furthermore, he continued to say that the quality of life is the individual's feeling of happiness and satisfaction with life through achieving a balance between the health, psychological, social and economic aspects and the degree of his/her compatibility with himself/herself and others. Likewise, Naissa (2012) argued that the quality of life is enjoyment to the physical conditions in the external 
environment, satisfying the needs, good sense and satisfaction with life, and the individual's awareness of aspects of his/her life in addition to his/her physical health and compatibility with the prevailing community values. Researchers argue that the quality of life is a positive psychological concept that includes satisfaction with life and enjoying. This concept includes various dimensions that define the quality of life for the individual, such as psychological and social health, self-fulfillment, and human needs.

According to (Tashtoush \& Al-Qashaar, 2017), the World Health Organization discussed the dimensions of the quality of life as follows:

- The physical dimension: It includes dealing with pain, discomfort, sleep and getting rid of fatigue.

- The psychological dimension: It consists of positive behaviors and feelings, the desire to learn, recalling and thinking, concentration, self-esteem, caring for one's appearance, and autonomy and negative feelings.

- The social dimension: It includes personal relationships, social support, successful marriage, and the special needs of the individual such as acceptance, interaction, and social loyalty.

- The environmental dimension: It includes exercising freedom, and the feeling of security and safety in the surrounding and home environment, income sources, and avoiding pollution and noise.

Abdel-Mu'ty (2005) also argued that there are three dimensions of the quality of life:

- The objective quality of life, i.e. what material resources the society provides its individuals with as well as the individual's social life.

- The subjective quality of life, which means the extent of personal satisfaction with life and the individual's perception of the quality of life.

- The existential quality of life, which represents the ideal level to satisfy the needs of the individual and the ability to live in a spiritual and psychological compatibility with one's self and community.

The current study focuses on physical health dimensions, psychological health, social relations and the environment to determine the level of quality of life for Individuals who Stutters.

\subsection{Quality of life for individuals who shutter}

Although several previous studies addressed the concept of the quality of life in general concerning several disorders and diseases, many of them ignored stuttering disorder, despite the fact that stuttering disorder is a communicative and social problem that has negative impacts not only on the emotional and psychological health but also on the lives of individual who Stutter.

One of the studies that addressed the importance of the quality of life and its dimensions among Individuals who Stutter is the study of Kasbi et.al. (2015) who divided the dimensions of the quality of life for stuttering adults into five dimensions, the most important of which are: Public health, emotional health, physical needs, social needs, vital emotional determinants, and pain, and they confirmed that one of the most important roles of a speech and language pathologist is an attempt to improve the quality of life of Individuals who Stutter by reducing functional and organic defects and determinants of communication and daily activities. Maree et.al. (2011) discussed the importance of guidance in improving the value of life design for adult Individuals who Stutter. The study adopted the quality of life approach for adult Individuals who Stutter as a case study. Various pre-strategies were used to engage with adults in discussions about the quality of life and the future they aspire for. After training, the study showed that the adult Individuals who Stutter are more able to make their own desired work decisions, especially with empowerment and personal agency.

Moreover, a group of researchers found that the impacts of the quality of life for adult Individuals who Stutter were often negative. Klompas and Ross (2004) studied the quality of life for adult Individuals who Stutter and found negative impacts of stuttering on emotions, self-esteem, self-image, and professional performance. The study of Kasbi et.al. (2015) addressed the impacts of stuttering on the quality of life of adult Individuals who Stutter. The results of the study showed that stuttering has negative impacts on the various dimensions of the quality of life scale among adult Individuals who Stutter. The study found statistically significant differences among groups concerning the quality of life scale in public and emotional health, physical and social functions resulted from emotional and physical problems. It is also found that there were no significant differences in the dimension of pain among the two groups. The results of the study of Craig (2009) related to the quality of the life of adult Individuals who Stutter and ordinary people showed that stuttering has negative impacts on emotional and social activities, psychological health, the more stuttering is frequent, the worse the emotional aspects are.

By reviewing the quality of the life of Individuals who Stutter and its relationship with some variables, Andrade et.al. (2014) found that simple and severe stuttering had negative impacts on the quality of life, whereas the study of Koedoot et.al. (2011) showed that medium and severe stuttering had negative impacts on all aspects of it. Similarly, Bramlett et. al. (2006) confirmed that stuttering has negative impacts on the quality of life, especially the intensity of stuttering. Moreover, Yaruss et. al. (2010) conducted a comprehensive evaluation for the experiences of speakers with stuttering before and after treatment taking into account that stuttering had negative impacts on the quality of life and showed that the quality of life increased after treatment. Concerning 
other variables, Mohammadi et. al. (2011) studied the quality of life among 59 Kurdish speakers and compared them to normal cases, who has no stuttering, in relation to the variables of gender, education, work, and social status using the World Health Organization Quality of Life scale- (BREF). They concluded that Individuals who Stutter' scores were less than their people who don't stutter according to the aspects of mental and physical health as well as social interactions, and that there was a negative correlation between the severity of stuttering and the dimensions of health, physical and mental environment.

Mansuri et. al. (2013) studied the quality of life in Tehran. The study sample consisted of (25) adult Individuals who Stutter and compared them to 25 people who don't stutter according to the variables of gender, age, and level of education using the World Health Organization Quality of Life scale (BREF). The results of the study indicated that there are significant differences on the overall measure of the quality of life and on the degrees of environmental, mental and physical aspects between adult Individuals who Stutter and non-Individuals who Stutter, while there are no differences attributed to the variable of social relations.

The study of Boyle (2015) aimed to investigate the relationship of social support, empowerment, and the quality of life self-help groups comprised of 240 Individuals who Stutter through a web-based survey. It was noted that increasing the level of self-esteem and the family social support are related to improving the quality of life of adult Individuals who Stutter regardless of the severity of stuttering. It was also found that consideration must be given to exercises that increase the feelings of self-esteem and strengthen the family social support for adult Individuals who Stutter in order to overcome stuttering and improve their quality of life.

Nang, et. al. (2018) studied the effect of stuttering on the development of autonomy, relationships, and the quality of life among women Individuals who Stutter. Given the fact that there are few studies on this subject especially that the prevalence of stuttering in males is higher than females. Study found that stuttering has extended impacts on all aspects of a woman's life as it greatly affects how she sees herself, her relationships, her profession capabilities and how others see her in society. As to the women who were interviewed, they showed a negative self-concept, and they felt that the level of their quality of life was affected by stuttering, and they considered that fluency was an essential factor in the development of personality and relationships. Therefore, the study recommended that speech and language specialists should take into consideration treatment plans and their impacts on the aspects of self-enhancement and the quality of life while training female Individuals who Stutter.

The study of Lucey et. al. (2019) aimed to find out whether there is a relationship between the temperament of adult Individuals who Stutter and the frequency of stuttering and its impact on the quality of life. The study found that the temperament of adult Individuals who Stutter is negative compared to ordinary individuals, and that adult Individuals who Stutter have frustration associated with a lack of knowledge of information about stuttering. It also found that there was no relationship between the feature of the temperament and the stuttering frequency and the quality of life.

Therefore, it is noteworthy that there are previous studies that addressed the impact of the quality of life and its correlation with some variables on Individuals who Stutter. Hence, the significance of the current study stems from the fact that it is the first study of its kind in the region - in researchers knowledge limits- since it comprises of Jordanian Individuals who Stutter and addresses the different dimensions that determine the level of the quality of life - physical health, psychological health, social relations, and the environment - according to a number of the most commonly important demographic variables such as age, gender, marital status, family income, and the degree of stuttering severity.

\subsection{Study problem}

Stuttering is one of the most common fluency disorders, and mainly affects the quality of life compared to other communication disorders as a Individuals who Stutter develops negative feelings, the inability to communicate smoothly and easily like others, thus, causing psychological pressure, tension, anxiety, and fear of any verbal situations. The quality of life problems is thus reflected on the psychological health, satisfaction, happiness, low self-esteem, the psychological development of an individual's personality, the feeling of deficiency, and the tendency to avoid others and move away from verbal situations that cause embarrassment. These feelings and frustrations could lead to slow or unsuccessful treatment and training that Individuals who Stutter undergo and, therefore, it is necessary to identify the most important factors that contribute to developing the positive energies of Individuals who Stutter enhance their attitude of themselves, and face verbal situations and life effectively.

In this regards, researchers, who investigate the stuttering disorder especially in Jordanian culture which considers -as many cultures- stuttering as a shame and deficiency sign for a person noticed that most of the programs designed to train individual Individuals who Stutter focus heavily on strategies for developing fluency, and overlook a large part of the focus on the psychological effects of stuttering, such as the effect of the quality of life on Individuals who Stutter. Therefore, there was an urgent need to conduct a study that addresses the concept the quality of life and its impacts on Individuals who Stutter to help them solve their verbal problems within various aspects.

Likewise, through the researchers' study of the literature review, they also noticed a clear and significant 
deficiency of studies in both the Jordanian and Arabic library databases related to the level of quality of life, especially among Individuals who Stutter. Therefore, this study attempted to provide a theoretical and reference framework for subsequent studies and for the speech and language specialists and others who deal with stuttering disorder to help them identify the most important factors that must be centre of concentration during training and preparing programs to improve the quality of life for Individuals who Stutter as one of the important drivers of success to overcome daily and psychological stuttering disorder problems. Hence, the study problem posed the following questions:

1.4.1 The first question

What is the level of quality of life for Individuals who Stutter in Jordan according to physical health, psychological health, social relationships and environment dimensions?

1.4.2 The second question

Are there statistically significant differences at the level of significance $(\alpha=0.05)$ in the quality of life of Individuals who Stutter attributed to:
a. Age b. Gender
c. Social status
d. Family income
e. The degree of stuttering

\subsection{Procedural definitions:}

\subsubsection{Quality of Life}

It represents the degree obtained by the Individuals who Stutter on the scale of the quality of life used in the study. 1.5.2 Individuals who Stutter

They are individuals who suffer from stuttering disorder in the intermediate and advanced age stages and who were enrolled in hearing and speech centres and clinics in Amman.

\section{Study limitations}

The generalization of the results of the study is determined according to the following:

- Time limitations: The study was conducted between September and March 2020.

- Spatial limitations: The study was applied in speech and hearing clinics in the capital city of Amman.

- Objective limitations: The generalization of the study results is determined according to the study scales used and within the criteria of its validity, reliability and study sample.

\section{Methods and procedures}

\subsection{Study population and sample}

The study population consisted of all clients of hearing and speech clinics in the capital city of Amman. There are (4) clinics that include (52) male and female clients diagnosed as having only stuttering by self-reports. After excluding the piloting sample of the survey, which consisted of (20) male and female clients., the final study sample consisted of (32) clients. The researchers distributed the study tool to them by hand and was recollected. The results of the study were statistically analyzed, and Table No. (1) shows the distribution of the study sample according to the personal variables:

Table 1 . The distribution of the study population according to the personal variables

\begin{tabular}{|l|l|l|l|}
\hline Variable & Variable category & Number & The ratio \\
\hline \multirow{3}{*}{ Age } & Intermediate (7-13 yrs.) & 7 & 21.9 \\
\cline { 2 - 4 } & Advanced (more than 14 yrs.) & 25 & 78.1 \\
\hline \multirow{4}{*}{ Degree of severity } & female & 19 & 59.4 \\
\cline { 2 - 4 } & male & 13 & 40.6 \\
\hline \multirow{3}{*}{ Marital status } & mild & 7 & 21.9 \\
\cline { 2 - 4 } & moderate & 8 & 25.0 \\
\cline { 2 - 4 } & Severe & 9 & 28.1 \\
\cline { 2 - 4 } & profound & 8 & 25.0 \\
\hline \multirow{3}{*}{ Family income } & single & 27 & 84.4 \\
\cline { 2 - 4 } & married & 5 & 15.6 \\
\cline { 2 - 4 } & divorced & - & - \\
\hline & Less 500 JDs & 13 & 40.6 \\
\cline { 2 - 4 } & $500-$ less 1,000 JDs & 11 & 34.4 \\
\cline { 2 - 4 } & More than 1,000 JDs & 25.0 \\
\hline
\end{tabular}

\subsection{Study tool}

To achieve the aims of the study, the Quality of Life Scale was used as follows:

The researchers referred to several quality of life scales, the most important of which is the Brief Quality of Life Scale - BRIEF-WHOQQL_ ( prepared by the World Health Organization (WHO), 2013). The survey consists of 
(100) items distributed on six domains, namely, physical health, psychological health, social relations, the environment, the level of autonomy, and the spiritual health, which was later abbreviated to (Brief-WOQOL) and consists of (26) items within four domains: physical health, psychological health, social relations, and the environment. This scale is used with ordinary, sick, and people with disorders (Skevington, S., Lotfy \& O 'Connell, K., 2004). The researchers also took advantage of a number of psychometric tools used to measure stuttering disorder, stuttering attitudes and their reactions to stuttering and the quality of life, including (Franic \& Bothe, 2008; Bramlett et.al., 2006; Abu Asaad, 2009; Al-Bdour, 2013; Tashtoush \& Al-Qashaar, 2017), to make adjustments in line with stuttering disorder on the (Quality of life scale- Brief scale-WHO, (BRIEF-WHOQQL)). The study tool was then finalized into (26) items distributed on four domains: physical health represented by items (1-7), the psychological health (8-15), the social relations (16-20), and the environment domain (21-26). Likert five-level scale (Very few, few, medium, large, and very large), where (1) is the assigned to very few and (5) to very large; reflected in the case of the following negative items (1), (13), (14), (19) and (20), where the highest score obtained by the respondent is (130) and the lowest is (26). Consequently, and for the purpose of this study, means were used to judge the level of quality of life for the respondents according to the following equation: the highest weight - the lowest weight divided by the number of categories, $5-1=4,4 / 3=1.33(5-1 \div 3=1.33)$, and accordingly, the total score is less than 2.33 and this indicates a low level of quality of life, and a score between 2.33- less than 3.67 indicates an average level of quality, 3.67 and above indicates a high level quality of life.

$>2.33=$ low level of quality of life

$2.33-\geq 3.67=$ average level of quality of life

3.67 and above $=$ high level quality of life

\subsection{Validity and reliability of the study tool}

\subsubsection{The validity and reliability of the study tool was verified by the following methods}

- Apparent validity (Reviewers):

The validity of the study tool was verified by (8) professors, at Al-Hussein Bin Talal University and the University of Jordan specialized in the fields of measurement and evaluation; psychology; and speech and language disorders, to express their opinion on the accuracy and validity of the scale content, clarity of items, study tool language, and the extent to which items are related to the domains and the scale. The reviewers made a number of observations that were taken into consideration, and $(80 \%)$ of the agreement between the reviewers was adopted.

- The validity of internal consistency:

The validity of the internal consistency of the study tool was verified by calculating the correlation coefficient between the domain and the total score and between the item and the domain to which it belongs and the total score, where the scale was applied to a pilot sample consisted of (20) males/ females samples of the study population and other sample. Table No. (2) shows the results: 
Table 2. Item's Correlation Coefficient with Domain Sample, Total Score, Domain and Total Score of The

Quality of Life Scale

\begin{tabular}{|c|c|c|c|c|c|c|}
\hline \multirow[t]{2}{*}{ Item } & \multicolumn{2}{|c|}{$\begin{array}{l}\text { Domain correlation with } \\
\text { total degree }\end{array}$} & \multicolumn{2}{|c|}{$\begin{array}{l}\text { Item correlation with total } \\
\text { degree }\end{array}$} & \multicolumn{2}{|c|}{ item correlation with domain } \\
\hline & P. Value & $\mathbf{R}$ & P. Value & $\mathbf{R}$ & P. Value & $\mathbf{R}$ \\
\hline & 0.000 & $* * 0.900$ & \multicolumn{4}{|c|}{ physical health domain } \\
\hline 1 & & & 0.012 & $* 0.551$ & 0.000 & $* * 0.761$ \\
\hline 2 & & & 0.000 & $* * 0.781$ & 0.000 & $* * 0.736$ \\
\hline 3 & & & 0.006 & $* * 0.592$ & 0.007 & $* * 0.580$ \\
\hline 4 & & & 0.000 & $* * 0.798$ & 0.000 & $* * 0.756$ \\
\hline 5 & & & 0.001 & $* * 0.667$ & 0.000 & $* * 0.814$ \\
\hline 6 & & & 0.000 & $* * 0.708$ & 0.000 & $* * 0.726$ \\
\hline \multirow[t]{2}{*}{7} & & & 0.017 & $* 0.528$ & 0.002 & $* * 0.655$ \\
\hline & 0.000 & $* * 0.937$ & \multicolumn{4}{|c|}{ psychological health domain } \\
\hline 8 & & & 0.001 & $* * 0.664$ & 0.001 & $* * 0.681$ \\
\hline 9 & & & 0.001 & $* * 0.695$ & 0.000 & $* * 0.797$ \\
\hline 10 & & & 0.001 & $* * 0.687$ & 0.000 & $* * 0.777$ \\
\hline 11 & & & 0.012 & $* 0.552$ & 0.011 & $* 0.554$ \\
\hline 12 & & & 0.000 & $* * 0.780$ & 0.000 & $* * 0.849$ \\
\hline 13 & & & 0.000 & $* * 0.705$ & 0.000 & $* * 0.761$ \\
\hline 14 & & & 0.000 & $* * 0.798$ & 0.000 & $* * 0.895$ \\
\hline \multirow[t]{2}{*}{15} & & & 0.000 & $* * 0.826$ & 0.000 & $* * 0.766$ \\
\hline & 0.000 & $* * 0.829$ & \multicolumn{4}{|c|}{ social relationships domain } \\
\hline 16 & & & 0.000 & $* * 0.739$ & 0.000 & $* * 0.858$ \\
\hline 17 & & & 0.007 & $* 0.581$ & 0.010 & $* 0.560$ \\
\hline 18 & & & 0.001 & $* * 0.690$ & 0.001 & $* * 0.705$ \\
\hline 19 & & & 0.001 & $* * 0.699$ & 0.000 & $* * 0.917$ \\
\hline \multirow[t]{2}{*}{20} & & & 0.041 & $* 0.461$ & 0.000 & $* * 0.724$ \\
\hline & 0.000 & $* * 0.853$ & \multicolumn{4}{|c|}{ environment' domain } \\
\hline 21 & & & 0.000 & $* * 0.857$ & 0.000 & $* * 0.744$ \\
\hline 22 & & & 0.000 & $* * 0.756$ & 0.000 & $* * 0.887$ \\
\hline 23 & & & 0.025 & $* 0.501$ & 0.000 & $* * 0.735$ \\
\hline 24 & & & 0.000 & $* * 0.793$ & 0.000 & $* * 0.877$ \\
\hline 25 & & & 0.001 & $* * 0.688$ & 0.000 & $* * 0.876$ \\
\hline 26 & & & 0.024 & $* 0.503$ & 0.002 & $* * 0.640$ \\
\hline
\end{tabular}

* Statistically significant at the level of $(\alpha \leq 0.05)$.

** Statistically significant at the significance level of $(\alpha \leq 0.01)$.

The results of Table (2) show that the correlation coefficients between the domain and the overall score ranged from (0.929-0.937). The item's correlation coefficients with the domain ranged (0.554-0.895), and the correlation coefficients of the item with the total score ranged between (0.461-0.857). This indicates that all correlation coefficients are statistically significant and thus the validity of the internal consistency of the study tool.

3.3.2 Tool reliability

The reliability of the study tool was verified using the internal consistency coefficients using the Cronbach's Alpha equation, and Table (3) illustrates the internal consistency factors of domains and the scale.

Table 3. Cronbach Alpha Internal Reliability Coefficients

\begin{tabular}{|l|l|l|l|}
\hline Number & domain & Items number & Reliability factor \\
\hline $\mathbf{1}$ & physical health & 7 & 0.773 \\
\hline $\mathbf{2}$ & psychological health & 8 & 0.880 \\
\hline $\mathbf{3}$ & social relationships & 5 & 0.787 \\
\hline $\mathbf{0}$ & environment & 6 & 0.882 \\
\hline
\end{tabular}

The results of Table (3) show that the coefficients of the Cronbach Alpha reliability for the domains ranged between (0.773-882) and for the scale as a whole (0.946) which means that they are appropriate values for conducting the study. 


\subsection{Study methodology}

The descriptive correlational approach was used to describe the reality of the level of the quality of life among Individuals who Stutter with disorder and to explain the nature of its relationship with some demographic variables related to the members of the study sample.

\subsection{Study variables}

\subsubsection{Independent variables}

- Age: It has two categories (intermediate stage (7- 13 years) and advanced stage (14 years or more).

- Gender: It has 2 categories (male and female).

- $\quad$ Stuttering severity: It has 4 levels (mild, moderate, severe, and very severe/ profound).

- Marital Status: It has 3 categories (single, married, separated)

- Family income: It has 3 categories (less than 500 JDs) (500-less than 1,000 JDs), (1,000 JDs and more) 3.5.2 Dependent variables: the level of the quality of life

\subsection{Statistical analysis:}

To achieve the aims of the study and to answer its questions, the following were used:

- $\quad$ 1. Pearson coefficients to verify the validity of the scale.

- 2. Cronbach Alpha coefficient to verify the scale reliability.

- 3. Means and standard deviations to answer the first and second study questions.

- 4. One-way ANOVA to verify whether the apparent differences were significant.

\section{Study results}

The results of the first study question "What is the level of quality of life for Individuals who Stutter in Jordan according to physical health, psychological health, social relationships and environment dimensions?

To answer this question, the means, standard deviations, rank, level of domains, and total score were calculated as shown in Table (4):

Table 4. Standard Deviations and Total Degree of Quality of Life of Individuals who Stutter in Jordan

\begin{tabular}{|l|l|l|l|l|l|}
\hline No. & Domain & mean & $\begin{array}{l}\text { Standard } \\
\text { deviation }\end{array}$ & Ratio & Level \\
\hline $\mathbf{1}$ & physical health & 2.23 & 0.537 & 1 & low \\
\hline $\mathbf{2}$ & psychological health & 1.95 & 0.481 & 2 & low \\
\hline $\mathbf{3}$ & social relationships & 2.06 & 0.591 & 3 & low \\
\hline $\mathbf{4}$ & environment & 1.92 & 0.549 & 4 & low \\
\hline Overall Quality of life & $\mathbf{2 . 0 4}$ & $\mathbf{0 . 4 3 1}$ & - & low \\
\hline
\end{tabular}

Table (4) shows that the overall mean of the level of the quality of life of Individuals who Stutter in Jordan is (2.04) with a standard deviation of (0.431), and this indicates a low degree of appreciation, and that the level of the quality of Individuals who Stutter' life is low and the domain of physical health ranked first with a mean of (2.23) and a standard deviation of (0.537), followed by the social relations with a mean of (2.06) and a standard deviation of (0.591), and in the third place the mental health with a mean of (1.95) and a standard deviation of (0.481), followed by the environmental domain with a mean of (1.92) and a standard deviation of (0.549).

For more declaration for the Means, standard deviations, rank, and level of the quality of life items among Individuals who Stutter in Jordan in the previous four domains, table (5) show the Means and standard deviations of physical health items.

Table 5. Means and standard deviations of the physical health items as one of the domains of the quality of life among Individuals who Stutter in Jordan

\begin{tabular}{|l|l|l|l|l|l|}
\hline No. & Domain & mean & $\begin{array}{l}\text { Standard } \\
\text { deviation }\end{array}$ & Ratio & level \\
\hline $\mathbf{1}$ & $\begin{array}{l}\text { I feel that stuttering prevents me from doing the work } \\
\text { I love }\end{array}$ & 2.88 & 1.211 & 2 & Moderate \\
\hline $\mathbf{2}$ & I can cope with stuttering disorder & 2.06 & 0.878 & 3 & low \\
\hline $\mathbf{3}$ & I feel good about my sleep & 1.69 & 0.896 & 7 & low \\
\hline $\mathbf{4}$ & I feel satisfied with my energy at work or study & 1.97 & 0.695 & 4 & low \\
\hline $\mathbf{5}$ & I am satisfied with my ability to perform daily activities & 1.96 & 0.782 & 5 & low \\
\hline $\mathbf{6}$ & I feel good about my health status & 1.72 & 0.813 & 6 & low \\
\hline $\mathbf{7}$ & I need a set of training sessions to fix my problem & 3.34 & 1.035 & 1 & moderate \\
\hline Total mean for physical health & $\mathbf{2 . 2 3}$ & $\mathbf{0 . 5 3 7}$ & - & low \\
\hline
\end{tabular}

The data of table (5) show that the overall mean of the physical health domain is (2.23) with a standard deviation of (0.537), and this indicates a low degree of appreciation, and that the physical health of Individuals 
who Stutter is low. Item (7) ranked first with a mean of (3.34) and a standard deviation of (1.035), followed by item (1) with a mean of (2.88) and a standard deviation of (1.211), then item (2) with a mean of (2.06) and a standard deviation of (0.878), and in the last place is item (3) with a mean of mean of (1.69) and a standard deviation of (0.896).

Table 6. Means and standard deviations of the psychological health domain as one of the domains of the quality of life among Individuals who Stutter in Jordan

\begin{tabular}{|l|l|l|l|l|l|}
\hline No. & Domain & mean & $\begin{array}{l}\text { Standard } \\
\text { deviation }\end{array}$ & Ratio & level \\
\hline $\mathbf{8}$ & I feel I have a high quality of life & 1.84 & 0.767 & 4 & low \\
\hline $\mathbf{9}$ & I enjoy my life & 1.66 & 0.553 & 7 & low \\
\hline $\mathbf{1 0}$ & I feel that my life has meaning & 1.72 & 0.729 & 5 & low \\
\hline $\mathbf{1 1}$ & I feel good about my outer body shape & 1.87 & 0.793 & 3 & low \\
\hline $\mathbf{1 2}$ & I Have the ability to focus & 1.72 & 0.683 & 5 & low \\
\hline $\mathbf{1 3}$ & $\begin{array}{l}\text { I feel negative emotions when speaking, such as anxiety, } \\
\text { depression and bad mood }\end{array}$ & 2.59 & 1.241 & 1 & moderate \\
\hline $\mathbf{1 4}$ & $\begin{array}{l}\text { I feel Shy from some behaviours while speaking, as } \\
\text { blinking of the eyes and shaking of the head or jaws }\end{array}$ & 2.53 & 0.950 & 2 & moderate \\
\hline $\mathbf{1 5}$ & I feel satisfy about myself & 1.69 & 0.644 & 6 & low \\
\hline $\mathbf{0 v e r a l l ~ m e a n ~ o f ~ p s y c h o l o g i c a l ~ h e a l t h ~}$ & $\mathbf{1 . 9 5}$ & $\mathbf{0 . 4 8 1}$ & & low \\
\hline
\end{tabular}

The data of table (6) show that the overall mean of psychological health among Individuals who Stutter is (1.95) with a standard deviation of (0.481). This indicates that there is a low degree of appreciation, and that the physical health level of Individuals who Stutter is low, where item (13) ranked first with a mean of (2.59) and a standard deviation of (1.241), followed in the second place by item (14), with a mean of (2.53) and a standard deviation of (0.950), and in third place, item (11) with a mean of (1.87) and a standard deviation of (0.793), whereas item (9) ranked last with a mean of (1.66) and a standard deviation of (0.553).

Table 7. Means and standard deviations of the social relations domain as one of the domains of the quality of life among Individuals who Stutter in Jordan

\begin{tabular}{|l|l|l|l|l|l|}
\hline No. & Domain & Mean & $\begin{array}{l}\text { Standard } \\
\text { deviation }\end{array}$ & Ratio & level \\
\hline $\mathbf{1 6}$ & I feel satisfied with my personal relationship & 1.75 & 0.672 & 4 & Low \\
\hline $\mathbf{1 7}$ & I am satisfied with the support I receive from my friends & 1.75 & 0.567 & 4 & Low \\
\hline $\mathbf{1 8}$ & I can deal good with the opposite gender & 2.16 & 0.677 & 3 & Low \\
\hline $\mathbf{1 9}$ & I feel mockery of many around me when I speak & 2.34 & 1.096 & 1 & Moderate \\
\hline $\mathbf{2 0}$ & I want to isolate from all around me & 2.31 & 1.256 & 2 & Low \\
\hline overall mean of social relationships & $\mathbf{2 . 0 6}$ & $\mathbf{0 . 5 9 1}$ & - & low \\
\hline
\end{tabular}

The data of table (7) show that the overall mean of social relations among Individuals who Stutter is (2.06) with a standard deviation of (0.591), and this indicates a low degree of appreciation, and that the level of social relations is a low, where item (19) ranked first with a mean of (2.34) and a standard deviation of (1.096), followed by item (20) with a mean of (2.31) and a standard deviation of (1.256), and in third place item (18) with a mean of (2.16) and a standard deviation of (0.677), followed by items (16) and (17) with a mean of (1.75) for each of them and a standard deviation of $(0.672)$ and $(0.567)$ respectively.

Table 8. Means and standard deviations of the environment domain as one of the domains of the quality of life among Individuals who Stutter in Jordan

\begin{tabular}{|l|l|l|l|l|l|}
\hline No. & Domain & mean & $\begin{array}{l}\text { Standard } \\
\text { deviation }\end{array}$ & Ratio & level \\
\hline $\mathbf{2 1}$ & I feel safe in my life & 1.63 & 0.609 & 5 & low \\
\hline $\mathbf{2 2}$ & I have the money that meets my needs & 2.19 & 0.931 & 1 & low \\
\hline $\mathbf{2 3}$ & I feel my environment is healthy & 1.91 & 0.856 & 4 & low \\
\hline $\mathbf{2 4}$ & $\begin{array}{l}\text { I have the information I need about stuttering disorder and } \\
\text { how to treat it }\end{array}$ & 2.13 & 0.871 & 2 & low \\
\hline $\mathbf{2 5}$ & I have the opportunity to rest and play leisure activities & 2.09 & 0.893 & 3 & low \\
\hline $\mathbf{2 6}$ & I feel good about my private life & 1.56 & 0.669 & 6 & low \\
\hline
\end{tabular}

The data of table (8) show that the overall mean of environment among Individuals who Stutter is (1.92) with a standard deviation of (0.549), and this indicates a low degree of appreciation, and that the level of environment is a low, where item (22) ranked first with a mean of (2.19) and a standard deviation of (0.931), followed by item (24) with a mean of (2.13) and a standard deviation of (0.871), and in third place item (25) with a mean of (2.09) 
and a standard deviation of (0.893), followed by item (26) with a mean of (1.75) for each of them and a standard deviation of (1.56) and standard deviation of (0.669).

The results of the second study question "Are there statistically significant differences at the level of significance $(\alpha \leq 0.05)$ in the quality of life of Individuals who Stutter attributed to: age, gender, degree of stuttering severity, marital status, and family income?"

To answer this question, One-Way ANOVA analysis of variance was used, and the following tables show the results:

Table 9. Means and standard deviations for the variables of age, gender, marital status, family income and degree of stuttering severity on a level of the quality of life.

\begin{tabular}{|c|c|c|c|c|c|c|}
\hline Variable & Variable category & number & mean & $\begin{array}{l}\text { Standard } \\
\text { deviation }\end{array}$ & $\begin{array}{l}\text { Modified } \\
\text { average }\end{array}$ & $\begin{array}{l}\text { Standard } \\
\text { error }\end{array}$ \\
\hline \multirow[b]{2}{*}{ age } & $\begin{array}{l}\text { Intermediate } \\
\text { (less13 yrs.) }\end{array}$ & 7 & 1.88 & 0.483 & 1.84 & 0.213 \\
\hline & $\begin{array}{l}\text { Advanced (more } 14 \\
\text { yrs.) }\end{array}$ & 25 & 2.08 & 0.415 & 1.98 & 0.120 \\
\hline \multirow{2}{*}{ gender } & male & 19 & 2.08 & 0.453 & 1.97 & 0.165 \\
\hline & female & 13 & 1.98 & 0.406 & 1.86 & 0.153 \\
\hline \multirow[b]{2}{*}{ Marital status } & single & 27 & 2.07 & 0.414 & 2.06 & 0.101 \\
\hline & married & 5 & 1.85 & 0.527 & 1.77 & 0.227 \\
\hline \multirow{3}{*}{$\begin{array}{l}\text { Family } \\
\text { income }\end{array}$} & less500 JDs & 13 & 1.99 & 0.495 & 1.90 & 0.183 \\
\hline & 500- less $1,000 \mathrm{JDs}$ & 11 & 1.98 & 0.442 & 1.66 & 0.197 \\
\hline & More 1,000 JDs & 8 & 2.20 & 0.294 & 2.18 & 0.194 \\
\hline \multirow{4}{*}{$\begin{array}{l}\text { Degree of } \\
\text { severity }\end{array}$} & mild & 7 & 1.92 & 0.436 & 1.75 & 0.193 \\
\hline & moderate & 8 & 2.15 & 0.419 & 2.18 & 0.242 \\
\hline & severe & 9 & 2.14 & 0.494 & 1.91 & 0.198 \\
\hline & profound & 8 & 1.92 & 0.386 & 1.80 & 0.194 \\
\hline
\end{tabular}

Results of table (9) show that there are apparent differences between the means of the study population concerning the level of the quality of life according to the age, gender, marital status, family income, and the degree of stuttering severity variables. In order to verify whether the differences were significant, One-Way ANOVA analysis of variance used as table (10) show the results:

Table 10. Results of One-Way ANOVA analysis of variance showing differences in the level of the quality of life Individuals who Stutter according to the age, gender, marital status, family income, and the degree of stuttering severity variables

\begin{tabular}{|l|l|l|l|l|l|}
\hline Source of contrast & $\begin{array}{l}\text { Sum of } \\
\text { squares }\end{array}$ & $\begin{array}{l}\text { Degrees of } \\
\text { freedom }\end{array}$ & $\begin{array}{l}\text { Average } \\
\text { squares }\end{array}$ & $\begin{array}{l}\text { Value of } \\
\text { (F) }\end{array}$ & $\begin{array}{l}\text { Statistical } \\
\text { significance }\end{array}$ \\
\hline age & 0.069 & 1 & 0.069 & 0.371 & 0.549 \\
\hline gender & 0.064 & 1 & 0.064 & 0.344 & 0.561 \\
\hline Marital status & 0.284 & 1 & 0.284 & 1.529 & 0.229 \\
\hline Family income & 0.734 & 2 & 0.367 & 1.978 & 0.161 \\
\hline Degree of severity & 0.499 & 3 & 0.166 & 0.896 & 0.458 \\
\hline error & 4.268 & 23 & 0.186 & & \\
\hline total & 138.892 & 32 & & & \\
\hline Overall corrected & $\mathbf{5 . 7 6 5}$ & $\mathbf{3 1}$ & & & \\
\hline
\end{tabular}

The results of table (10) show that there are no statistically significant differences in the level of the quality of life among Individuals who Stutter in Jordan according to age, gender, marital status, family income and the degree of stuttering severity depending on the calculated (F) values at the level of probability accompanying it, and that they are not statistically significant at the level $(\alpha \leq 0.05)$.

\section{Discussion}

The results of the study indicated that the level of the quality of life among Individuals who Stutter in Jordan was low and the researchers attributed this decrease to the negative impacts that result from stuttering disorders and that they reduce the extent of human satisfaction with one's self in various aspects. Moreover, the physical health domain ranked the first lowest level of the quality of the life of Individuals who Stutter, and specifically for the item that includes the need for more training sessions to treat the problem. Many Individuals who Stutter develop physical behaviors that are not nature or involuntary, such as eye blinking, head, jaw, or body nodding as a reaction to hide the main behaviors of stuttering - repetition, blocks and prolongation - and, therefore, most of their problems were the need for training sessions to get rid of these behaviors and physical features. The second lowest 
domain was the social relations specifically for the item of a feeling of contempt for those who mocked Individuals who Stutter when speaking, and the researchers attributed this to the fact that human communication is a social need in the first place and among its main components is fluency and, thus, the disfluency or its problems greatly affect the extent of the interaction between an individual and his/her surroundings. Moreover, the repeated failures to communicate with others generate feelings of distance, isolation, and a tendency to avoid mockery of others. The third lowest domain was the psychological health, specifically for the item that shows Individuals who Stutter develop negative feelings such as anxiety and depression when speaking. This is due to the role of the general psychological side in the life of Individuals who Stutter, his/her lack of acceptance of the problem of stuttering, the weak satisfaction with his/her verbal behavior, the negative feelings, anxiety, and depression that the Individuals who Stutter develops while speaking.

The last lowest domain was the environment, specifically the item related to the material aspect of not having enough money to meet the needs of the Individuals who Stutter. The researchers attributed the low quality of life in the environment domain because the environment surrounding Individuals who Stutter does not support and accept the difference in speech between Individuals who Stutter and ordinary individuals, especially with the emergence of material problems including the need for training and guidance sessions, and psychological and social support in order to adapt to the surrounding environment and get rid of the problem. The results of the current study concorded with the study of Kasbi (2015), Craig (2009), Klompass (2004), and Merre (2011) who found that stuttering negatively affects the quality of life, especially in the emotional and social aspects.

The results of the study also showed that there are no statistically significant differences in the level of the quality of life according to a number of variables, the first of which is the age variable, where the quality of life level for each of the intermediate stage (13-6 years) and the advanced (14 years and older) appeared low, and the researchers attributed this to the fact that Individuals who Stutter develop more negative feelings and attitudes towards themselves as they get older and affect their satisfaction with their lives, and develop avoidance behaviors to reduce their stuttering occurrences and make them overcome such stuttering. Nevertheless, they develop great psychological pressures that affect the quality of life. These results differed with those of the study of Mansurre (2013) which found differences on the mental and physical dimensions of Individuals who Stutter according to the age variable.

The results also indicated that there are no statistically significant differences in the level of the quality of life according to the gender variable. They also indicated that the level of the quality of life for both males and females was low. The researchers attributed that despite the fact that many studies have confirmed that the occurrences of stuttering disorder among males have a higher rate than females, both males and females have problems in education, training, care and openness to the surrounding community, and suffer from a lack of opportunities for social and language interaction as a result of verbal disorder. Moreover, the results of the study concorded with Nang's (2018) study which focused on that stuttering affects all aspects of women's life.

Furthermore, the results of the current study indicated that there are no statistically significant differences in the level of the quality of life according to the variable of the marital status, as both the single and married have problems and a decrease in the quality of life. The single individual suffers from problems of emotional stability and the obsession of forming a family, as well as the married person suffers from problems, connections, needs, pressures, and some restrictions imposed by the nature of marriage including many social and financial relationships. The results concorded with Boyle's (2015) and Mohandi's (2011) studies, which emphasized the importance of the role of the family and self and social support groups in improving the quality of life.

In addition to this, the results showed that there are no statistically significant differences according to the family income or economic situation in the level of the quality of life of Individuals who Stutter regardless of their low, medium, or high incomes. All of them suffer from financial and economic problems that negatively affect the life of the individual, create a sense of the lack of satisfaction and stability and the difficulty of taking training and rehabilitation sessions, which are often costly. Therefore, Individuals who Stutter have less chance of improvement and recovery from disorders, thus, leading to a lower level of quality of life, particularly in the environmental domain. The results of the current study concorded with those of Yaruss' (2011) which emphasized the improvement in the quality of life after treatment and training.

Finally, concerning the degree of stuttering severity variable, the results showed that there are no statistically significant differences in the low level of the quality of life (mild, moderate, severe, and very severe/profound degrees). The researchers attributed this to the problems and symptoms experienced by Individuals who Stutter including fear, anxiety, and avoidance of verbal situations. However, this degree of severity varies and develops and consequently the quality of life is affected and decreased regardless of the degree of stuttering severity. Moreover, the results of the study differed from those of Kodot's study which emphasized that the moderate stuttering degree highly affects the quality of life, and concorded with the studies of Braraellet (2006), Andrade (2008), and Mohamdi, et.al. (2011) who confirmed that the more severe stuttering, the more the level of the quality of life of Individuals who Stutter is affected negatively. 


\section{Conclusion and recommendations}

The study examined an important psychological aspect, which is the concept of the quality of life in its various dimensions, and its impact on the most common fluency disorders, stuttering disorder, and its relationship to some important demographic variables such as age, gender, marital status, family income, degree of stuttering severity. The concept of the quality of life is a contemporary concept addressed by several recent studies and tackles with the extent of the individual's satisfaction with his/her life in several aspects. It was found that the World Health Organization (WHO) focused on this concept and developed measures for a number of diseases and disorders to shed light on this aspect that greatly affects the concept of self-esteem and psychological well-being for individuals, as those with stuttering disorder, and contributes significantly to increasing the extent of improvement in treatment and training.

The results of the study showed that the level of the quality of life of Individuals who Stutter in Jordan was not satisfactory but rather low, especially the domain related to the lower physical dimensions. Stuttering disorder, according to the researchers, is a problem in the quality of life in the first place, and negatively and significantly affects several areas and aspects of Individuals who Stutter, such as social relations, psychological health, and the environment.

The quality of life of Individuals who Stutter did not differ statistically according to several variables, consequently, the level of the quality of life was low regardless of ag (advanced, intermediate) or gender (male, female) or marital status (single, married and separated) or economic income (high, medium and low) or stuttering severity ( mild, moderate, severe, and very severe/ profound).

Accordingly, the researchers recommend a set of recommendations that would enhance the level of the quality of life of Individuals who Stutter, the most important of which are:

- Creating self-support, and community and family support groups of Individuals who Stutter.

- The training programs should include strategies to enhance the dimensions of the physical, psychological, and social concept, and environment.

- Preparing a guidebook in cooperation with the World Health Organization that includes the most important dimensions of the concept of the quality of life speech and language pathologist need to assess and train of Individuals who Stutter.

- Conducting further studies and research related to the psychometric measures of the quality of life of Individuals who Stutter.

- Promoting positive attitudes and developing the quality of life concept programs of Individuals who Stutter.

\section{References}

Abdel-Mu'ty, H. (2005). Psychological counseling and quality of life in contemporary society. A paper submitted to the third conference for the psychological and educational development of the Arab in the light of the quality of life. Al-Zaqeeq University: Egypt. 13-23.

Abu Asaad, A. (2009). Guide for standards and psychological and educational tests. Amman. Jordan: Dino for Printing and Publishing.

Abu Rasin, M. (2012). The effectiveness of a proposed training program to improve the quality of life for educational diploma students at King Khalid University. Journal of Psychological Counseling. Ain Shams University (30). 233-187.

Al-Bdour, N. (2013). The effectiveness of a training program based on neuro-linguistic theory in reducing stuttering behavior among a sample of Jordanian children. (Unpublished doctoral dissertation). International Islamic Science University, Amman, Jordan. Educational Sciences. 13(2), 133-151.

Al-Khatib, J., Al-Smadi, J., Al-Rousan, F., Al-Hadidi, M., Yaji, K., Al-Natour, M., Al-Surour, N. (2018). An introduction to the education of students with special needs. Amman: Jordan. Dar Al-Fikr for Publishing and Distribution. 8.

Al-Zahir, Q. (2010). Speech and language disorders. 1st edition. Amman. Jordan: Wael House for Publishing and Distribution.

Al-Zureikat, (2018). Speech and language disorders, diagnosis and treatment. 4th edition. Amman. Jordan: Dar Al-Fikr for Publication and Distribution.

Amayreh, M. \& Natour, Y. (2014). Introduction to communication disorders. 2nd edition. Amman. Jordan: Dar AlFikr for Publication and Distribution.

Andrade, C., Cunha, M., Juste, F., Ritto, A., \& Almedia, B. (2014). Self-perceptions of people who stutter regarding their experiences and results of stuttering treatments. CoDAS. 26(5), 415-420.

Bloodstein, O. \& Bernstein, R., N. (2008). A handbook on stuttering. 6th ed. Clifton Park, NY: Thomson Delmar learning.

Boni, A., Patric, D. \& Bushnell, D. (2002). Validation of united stat version of world health organization quality of life. Journal of Clinical Epidemiology. 53(1), 1-12. 
Boyle, M. P., Beita, C., Milewski, K. \& Fearonm, A. (2017). Self -esteem, self -efficacy and social support as predictors of communicative participation in adults who stutter. Journal of Speech, Language and Hearing Research. 61, 1893-1906.

Boyle, M. (2015). Social support, self-concept prove key for adults who stutter. The ASHA leader.

Bramellett, R., Bothe, A. \& Franic, D. (2006). Using preference- based measures to assess quality of life in stuttering. Journal of Speech, Language and Hearing Research. 49, 381-394.

Byrd, C. T., McGill, M., Gkalitsiou, Z. \& Cappellini, C. (2017). The effects of self- disclosure on male and female perceptions of individuals who stutter. American Journal of Speech -Language Pathology. 26, 69-80.

Cherif, L., Boudabous, J., Khammoun, K., Kammoun, S., Kaceam, I. \& Moalla, Y. (2018). Self-esteem and anxiety in stuttering children an attitude of their parents. Health Edu Care. 3(2), 1-5.

Craig A, Blumgart E, T Y. 2009.The impact of stuttering on the quality of life in adults who stutter, J Fluency disorder.34(2).61-71.

Damico, T., Muller, N. \& Ball, M. (2010). The handbook of language and speech disorders. Wiley: Black Well Publication

Ellen, M., B. (2006). Working with people who stutter: A lifespan approach. Pearson Merrill, Prentice Hall.

Franic, M. D. \& Bothe, A. K. (2008). Psychometric evaluation of condition specific - instruments used to assess health related quality of life, attitudes and related constructs in stuttering. American journal of speech language pathology. 17, 60-80.

Graham-Bethea, J.\& Mayo, R. (2012). Perspective: A minority within a minority. The ASHA leader. 17(2), 20121.

Guitar, B. (2006). Stuttering: An integrated approach to its nature and treatment. 3rd ed. Lippincott Williams \& Wilkins, Philadelphia University.

Guttormsen, L S.(2018). A multimethod study of the impact of stuttering on children. A Dissertation for the degree of Philosophiae Doctor (PhD). Faculty of Educational Sciences, University of Oslo.

Halfond, M., Gottwald, \& Starkwather, W. (1990). A clinical method stuttering prevention. Prentice Hall.

Kasbi, F., Mokhlesin, M., Maddah, M., Noruzi, R., Noruzi, M., Leyla \& Khani, M. (2015). Effects of stuttering on quality of life in adults who stutter. Middle East Health. 2 (1), 1-5.

Kathard, H., Norman, V., \& Pillay, M. (2010). Configurations of self -identity formations of adults who stutter. SAJCD. 57, 51-66

Katloo, K., \& Tayseer, A. (2011). Quality of life and its relationship to mental health. Journal of Psychology. 24 (88), 89-64.

Klompas, M; Ross,E. 2004. Life experience of people who stutter and the perceived impact of stuttering on quality of life, Personal accounts of south African individual. J fluency disorder. 29, (4). 275-305.

Koedoot C, Bouwmans C, Franken MC,Stolk E. 2011. Quality of life in adults who stutter. J Communication Disorder. 44 (4):429-443.

Lucey, J., Evans, D. Maxfiel. (2019). Temperament in adults who stutter and its association with stuttering frequency and quality of life impacts. Journal of speech, language and hearing research. 62, 2691-2702.

Mansuri B, Shahbodaghi MR,Tohidast SA,Kamali M. 2013. Comparing the quality of life in adults who stutter with their normal counterparts. J research rehabilitation. 9(2):318-32

Maree, J., G., \& Hancke, Y. (2011). The value of life design counselling for an adolescent who stutter. Journal of Psychology in Africa. 21(3), 479-486.

Mohammadi H, Rezaei M , Moradi S, Barani M, Badri S,Heydari A. 2011.Comparison of quality of life in stuttering people with normal people. J Kermanshah UMS. 16(6):480-6.

Naissa, R. (2012). Quality of life for Damascus and Tishreen University students. Damascus University Journal. 21(1).

Nang, C., Hersh, D., Milton, K. \& Lau, S., L. (2018). The impact of stuttering on development of self - identity, relationships, and quality of life in women who stutter. American journal of speech -language pathology. 27, $1244-1258$

Ramig, P. R., \& Dodge, D. (2010). The child and adolescent stuttering treatment and activity resource guide. Clifton Park, NY: Delmar Cengage Learning.

Sheehan, J., G. (1970). Stuttering research and therapy. Harper \& Row, Michigan University.

Skevington, S., Lotfy, \& O' Connell, K. (2004). The World Health Organization's WHOQOL-BREF quality of life assessment: Psychometric proprieties and results of the international field trial: A Report from the WHOQOL Group. Quality of life research. 13, 299-310.

Tashtoush, R., \& Al -Qashaar, M. (2017). Quality of life and self-esteem among diabetics in Jordan. Jordanian Journal of Educational Sciences. 2(13), 133-15.

Yarus JS.2010. Assessing quality of life in stuttering treatment outcomes research. J Fluency Disorder. 35(3): 190202. 